\title{
Scintillation of Venus and Mars Express radio signal on interplanetary and ionosphere plasma
}

\author{
Guifré Molera Calvés* \\ Joint Institute for VLBI in Europe \\ Aalto Universtiy Metsähovi Radio Observatory \\ E-mail: molera@jive.nl
}

\section{Sergei Pogrebenko}

Joint Institute for VLBI in Europe

E-mail: pogrebenko@jive.nI

\section{Giuseppe Cimò}

Joint Institute for VLBI in Europe

E-mail: cimo@jive.nI

\section{Dmitry Duev}

Joint Institute for VLBI in Europe

E-mail: duev@jive.nI

\section{Tatiana Bocanegra-Bahamón}

Joint Institute for VLBI in Europe

E-mail: bocanegra@jive.nI

\section{Kamlesh Rajpurohit}

Thüringer Landessternwarte

E-mail: phy .kamlesh@gmail .com

\begin{abstract}
Observations of spacecraft radio signals give a unique opportunity to study the temporal and spatial behaviour of the signal's phase fluctuations caused by its propagation through the interplanetary plasma and the Earth's ionosphere. The phase scintillation of the telemetry signal of the European Space Agency's (ESA) Venus Express (VEX) and Mars Express (MEX) spacecraft was observed at X-band with a number of radio telescopes of the European VLBI Network (EVN) in the period 2008-2014.

It was found that the phase scintillation spectra follow a Kolmogorov distribution with nearly constant spectral index of -2.42 for a full range of orbital phases. The scintillation index and Doppler noise along the orbit from superior conjunction to the greatest elongation is dominated by the solar wind plasma. Here, I will present the latest results of these observations, while Venus is approaching the inferior conjunction, and where the Earth ionosphere starts to dominate.
\end{abstract}

12th European VLBI Network Symposium and Users Meeting

7-10 October 2014

Cagliari, Italy

* Speaker. 


\section{Introduction}

Observations of spacecraft radio signals within the solar system give a unique opportunity to investigate several science cases. In this work, we focussed on studying the temporal and spatial behaviour of the signal's phase fluctuations caused by its propagation through the interplanetary plasma and the Earth's ionosphere. The phase scintillation of the telemetry signal of the European Space Agency's (ESA) Venus Express (VEX) and Mars Express (MEX) spacecraft was observed at X-band with a number of radio telescopes in the period 2008-14. These measurements can be applied to a wide range of research fields, such as, the study of the gravity field of Phobos with Mars Express fly-by [Molera Calvés, 2012], study of solar wind turbulences using precise analysis of spacecraft phase [Molera Calvés et al., 2014], study of drag effect on VEX spacecraft in the atmosphere of Venus [Rosenblatt et al., 2012], and precise estimate of spacecraft state vectors of Venus Express [Duev et al., 2012].

\section{Methodology}

Our team initiated systematic observations of planetary spacecraft in 2009. ESA's VEX spacecraft was selected due to its high quality signal, suitable transmission frequency and possibility to observe with European VLBI radio telescopes. The observations lasted 5 years until VEX stop operations at the ends of 2014. Observations of ESA's MEX spacecraft started in parallel in 2013. MEX is the primary target for current and future observations.

A total of 317 observations with VEX data and 147 with MEX data have been conducted until the ends of 2014. These observations have been carried out with 20 different VLBI radio telescopes. Table 1 lists the main characteristics of these antennae.

The goal was to cover as well as possible the full orbits of Venus and Mars around the Sun. We achieved a good coverage of the Venus orbit thanks to the five years of data. So far, we have collected data for only a quarter of the orbit of Mars. The left image of Figure 1 shows the geometrical position of the observations conducted. The right image shows a sketch of the observations.
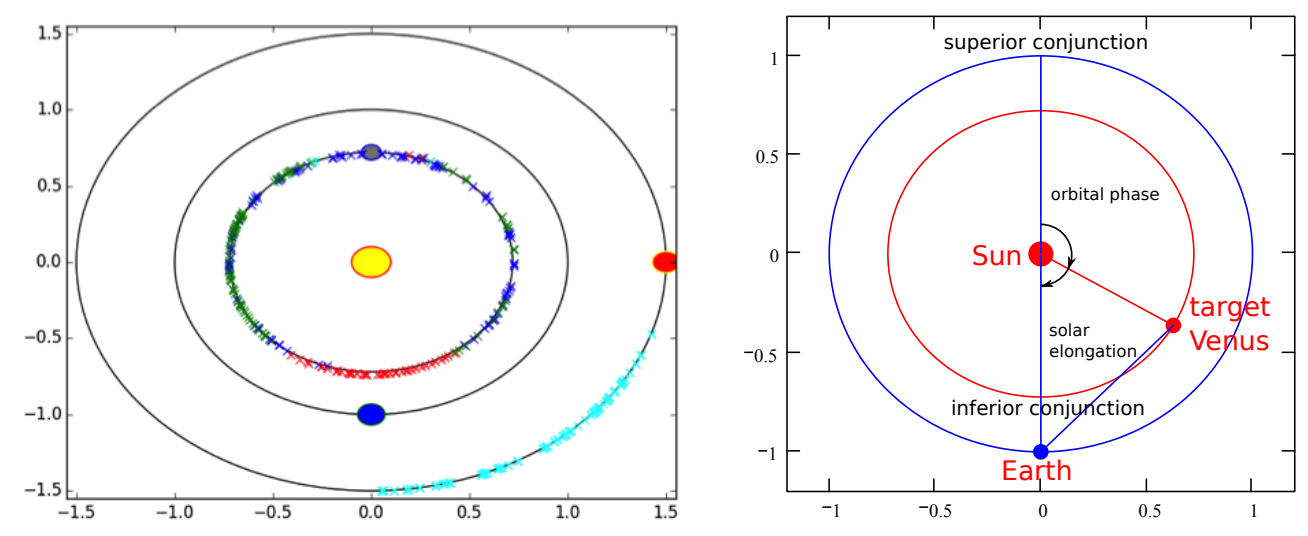

Figure 1: Left: observations conducted with respect of the position of the target with the Sun and Observer. Venus data have been divided into three different colours to differentiate time of observation. Right: sketch of the observations. 
Table 1: Specifications of the radio telescopes: antenna name, station code, country, latitude, longitude, elevation [m], and antenna diameter $(\varnothing)[\mathrm{m}]$.

\begin{tabular}{lcccccc} 
Antenna & Code & Country & Latitude & Longitude & Elev. & $\varnothing$ \\
\hline \hline Badary & $\mathrm{Bd}$ & $\mathrm{RU}$ & $102^{\circ} 14^{\prime} 02^{\prime \prime}$ & $51^{\circ} 46^{\prime} 13^{\prime \prime}$ & 822 & 32 \\
Hartebeesthoek-26 & $\mathrm{Hh}$ & $\mathrm{ZA}$ & $-25^{\circ} 53^{\prime} 14^{\prime \prime}$ & $27^{\circ} 41^{\prime} 05^{\prime \prime}$ & 1415 & 26 \\
Hartebeesthoek-15 & $\mathrm{Ht}$ & $\mathrm{ZA}$ & $-25^{\circ} 53^{\prime} 23^{\prime \prime}$ & $27^{\circ} 41^{\prime} 04^{\prime \prime}$ & 1374 & 15 \\
Kunming & $\mathrm{Km}$ & $\mathrm{CN}$ & $2^{\circ} 01^{\prime} 38^{\prime \prime}$ & $102^{\circ} 47^{\prime} 45^{\prime \prime}$ & 1973 & 40 \\
Matera & $\mathrm{Ma}$ & $\mathrm{IT}$ & $4^{\circ} 38^{\prime} 58^{\prime \prime}$ & $16^{\circ} 42^{\prime} 14^{\prime \prime}$ & 543 & 20 \\
Medicina & $\mathrm{Mc}$ & $\mathrm{IT}$ & $4^{\circ} 31^{\prime} 14^{\prime \prime}$ & $11^{\circ} 38^{\prime} 49^{\prime \prime}$ & 67 & 32 \\
Metsähovi & $\mathrm{Mh}$ & $\mathrm{FI}$ & $60^{\circ} 13^{\prime} 04^{\prime \prime}$ & $24^{\circ} 23^{\prime} 25^{\prime \prime}$ & 75 & 14 \\
Noto & $\mathrm{Nt}$ & $\mathrm{IT}$ & $36^{\circ} 42^{\prime} 34^{\prime \prime}$ & $14^{\circ} 59^{\prime} 20^{\prime \prime}$ & 143 & 32 \\
Onsala & $\mathrm{On}$ & $\mathrm{SE}$ & $5^{\circ} 23^{\prime} 47^{\prime \prime}$ & $11^{\circ} 55^{\prime} 39^{\prime \prime}$ & 10 & 20 \\
Pushchino & $\mathrm{Pu}$ & $\mathrm{RU}$ & $37^{\circ} 37^{\prime} 53^{\prime \prime}$ & $54^{\circ} 49^{\prime} 20^{\prime \prime}$ & 180 & 22 \\
Sheshan & $\mathrm{Sh}$ & $\mathrm{CN}$ & $31^{\circ} 05^{\prime} 57^{\prime \prime}$ & $121^{\circ} 11^{\prime} 58^{\prime \prime}$ & 29 & 25 \\
Svetloe & $\mathrm{Sv}$ & $\mathrm{RU}$ & $2^{\circ} 46^{\prime} 55^{\prime \prime}$ & $60^{\circ} 31^{\prime} 56^{\prime \prime}$ & 86 & 32 \\
Urumqi & $\mathrm{Ur}$ & $\mathrm{CN}$ & $43^{\circ} 28^{\prime} 17^{\prime \prime}$ & $87^{\circ} 10^{\prime} 41^{\prime \prime}$ & 2033 & 25 \\
Wettzell & $\mathrm{Wz}$ & $\mathrm{DE}$ & $4^{\circ} 08^{\prime} 42^{\prime \prime}$ & $12^{\circ} 52^{\prime} 03^{\prime \prime}$ & 670 & 20 \\
Yebes & $\mathrm{Ys}$ & $\mathrm{ES}$ & $40^{\circ} 31^{\prime} 27^{\prime \prime}$ & $-3^{\circ} 05^{\prime} 22^{\prime \prime}$ & 999 & 40 \\
Zelenchukskaya & $\mathrm{Zc}$ & $\mathrm{RU}$ & $4^{\circ} 33^{\prime} 54^{\prime \prime}$ & $43^{\circ} 47^{\prime} 16^{\prime \prime}$ & 1175 & 32
\end{tabular}

The interplanetary plasma total electron content (IP TEC) is calculated as it was described in [Molera Calvés et al., 2014], using a normal electron density of $5 \mathrm{~cm}^{-3}$ at 1 AU [Axford, 1968]. However, to recreate a more accurate environment we also compare the data with IP TEC in cases of fast and slow solar winds models [You et al., 2012].

The contribution of the Earth ionosphere is estimated using the vertical TEC maps provided by the International GNSS Service (IGS) ${ }^{1}$ based on global positioning satellites data. We modelled the ionosphere as a single model and estimate de vTEC values on a global grid with a spatial resolution of $5^{\circ}$ in longitude and $2.5^{\circ}$ in latitude in two-hour temporal resolution. As both VEX and MEX operate in two-way link mode, we take into account the contribution to the ESA's transmission station (uplink) and to the reception at our antenna (downlink).

The data processing is conducted with the software developed on-purpose for multi-tone tracking of spacecraft signals: SWspec, SCtracker and digital PLL. The software is described in detail in [Molera Calvés, 2012]. The three most important parameters out of our detections are the Doppler noise of the the detection, the carrier SNR ratio and the phase scintillation indices.

\section{Results}

In this paper, we present the results obtained with the observations of the two satellites orbiting Venus and Mars. It was found that the phase scintillation spectra follow a Kolmogorov distribution [Kolmogorov, 1991] with nearly constant spectral index of $-2.42 \pm 0.25$. The spectra follows a Kolmogorov distribution regardless its location on superior, inferior or greatest elongation with

\footnotetext{
${ }^{1} \mathrm{ftp} / / /$ cddis.nasa.gov/pub/gps/products/index
} 
respect to the Sun. On the other hand, the phase scintillation indices $\left(\sigma_{S c}\right), r m s$ of the phase fluctuations, depend on the propagation along the interplanetary media. The phase scintillation indices with respect to both, the orbital phase and the solar elongation are presented in Figure 2. In this example, only data collected from VEX spacecraft is presented, up to a maximum solar elongation of $45^{\circ}$.
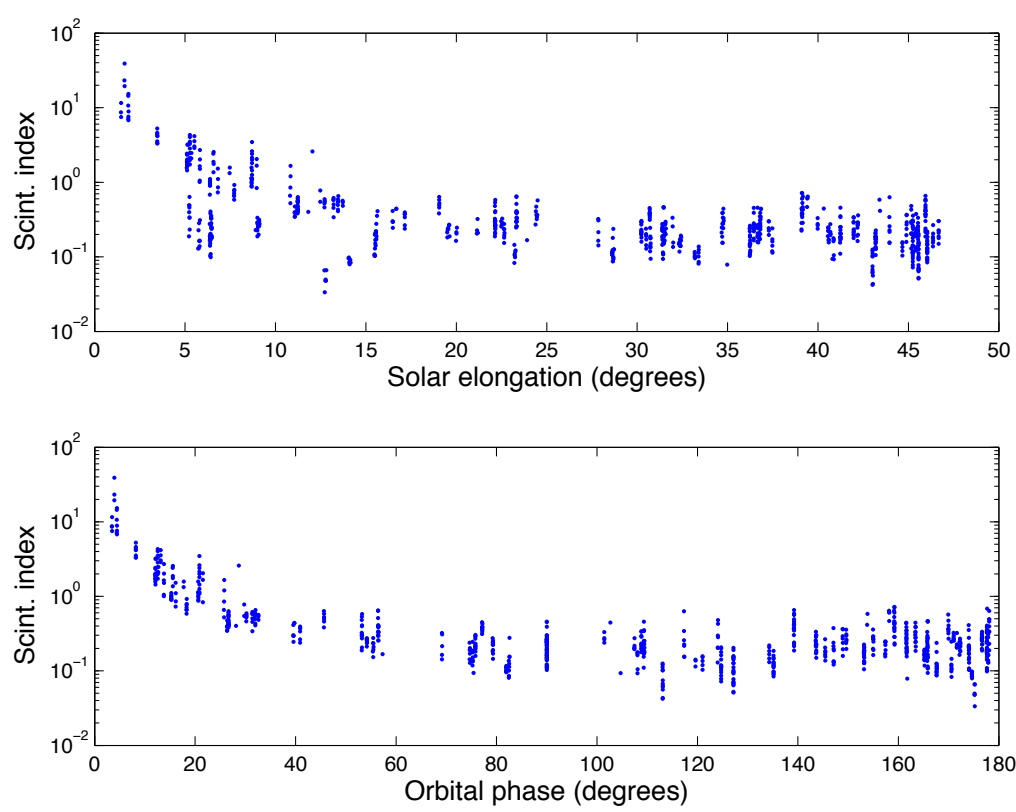

Figure 2: Phase scintillation index with respect to the solar elongation (top panel), in which the data exhibits a confusing ambiguity at low angles. Phase scintillation index with respect to the orbital phase (bottom panel) improves the display of the results.

The upper panel in Figure 2 shows the phase scintillation index with respect to the solar elongation. Part of these results were already published in [Molera Calvés et al., 2014]. The results observed during 2014 have been included here. Total data-set covers almost a full range of orbital phases, starting from $5^{\circ}$, near the superior/inferior conjunction, to $45^{\circ}$, at the greatest elongation. The indices values cover the range from 0.04 to 40 radians in the scintillation band $(3 \mathrm{mHz}$ to $3 \mathrm{~Hz}$ wide).

The panel below of Figure 2 shows the scintillation index distribution with respect to orbital phase for the same period. The orbital phase allows us to display more clear the indices results. The flattening of the scintillation index at low values of interplanetary plasma TEC, near the inferior conjunction, is clearly seen in this graph.

Several factors contributed to the measured $\sigma_{S c}$ : the Allan variance of $\mathrm{H}$-maser clocks, the ionospheric TEC, the tropospheric contribution, or the instrumentation effect. With the available data, we disentangle the contribution of all these factors in our measurements.

$$
\sigma_{S c}^{2}=\left(\mathrm{TEC}_{i p} \cdot \mathrm{Sc}_{i p}\right)^{2}+\left(\mathrm{TEC}_{i o} \cdot \mathrm{Sc}_{i o}\right)^{2}+\left(\mathrm{K}_{t r} \cdot \mathrm{Air}_{m}\right)^{2}+\mathrm{PCal}^{2}+\mathrm{Bpr}^{2}
$$

Each contribution is well described in Table 2. 
Table 2: Contribution of several factors in the measured phase scintillation.

\begin{tabular}{l|l} 
& \\
\hline$\sigma_{S c}^{2}$ & measured scintillation index in radians \\
$\mathrm{TEC}_{i p}$ & interplanetary two-way TEC (tecu) \\
$\mathrm{TEC}_{i o}$ & ionospheric two-way TEC (tecu) \\
$\mathrm{Sc}$ & scaling factor for IP and Ionospheric TEC, radians per tecu \\
$\mathrm{Air}_{m}$ & two-way airmass, in units of $1 \mathrm{~kg} / \mathrm{cm}^{2}$ \\
$\mathrm{PCal}$ & instrumental phase error, derived from the Phase Cal analysis \\
$\mathrm{Bpr}$ & base phase variation, due to Allan variance of Tx and Rx H-maser clocks \\
$\mathrm{K}_{t r}$ & scaling coefficient for troposphere, radians per unit of airmass
\end{tabular}

The phase scintillation index with respect to the orbital phase, the contribution of the ionospheric TEC for the up- and downlink (station-to-spacecraft and spacecraft-to-station), the troposphere and instrument ion effect are shown in Figure 3.

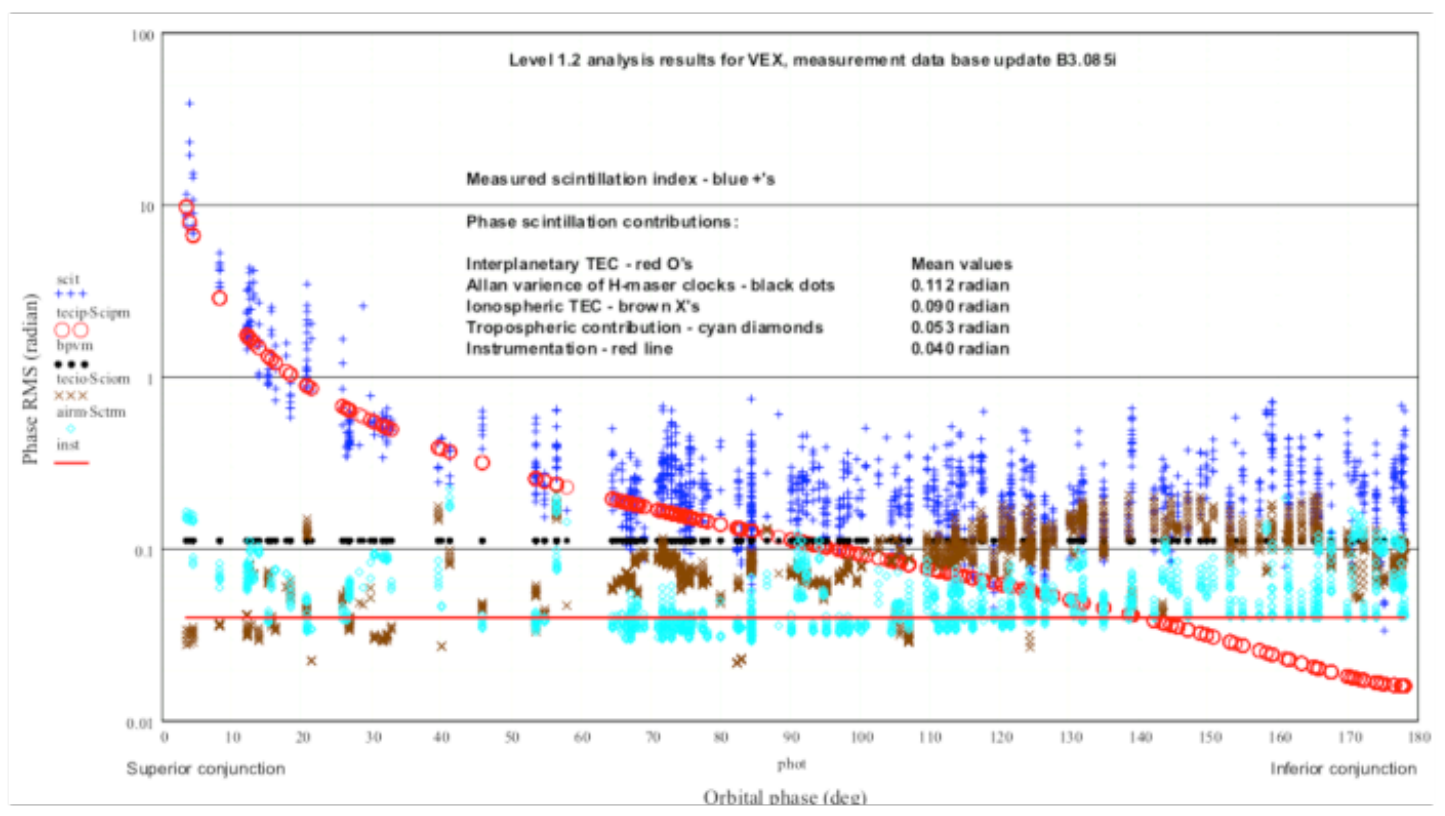

Figure 3: Interplanetary TEC (red circles), ionospheric TEC (brown crosses), Allan variance of the H-maser clocks (black dots), tropospheric contribution (cyan diamonds) and instrumentation error (red line).

Figure 4 projects the measurements estimated from the MEX data. As seen in Figure 1, the orbit coverage of MEX is lower, and we have lower data points than in Figure 3.

\section{Discussion and conclusions}

We have successfully demonstrated the accuracy of the VLBI phase-referencing method for tracking spacecraft in a number of occasions [Duev et al., 2012, Duev et al., 2015]. The nodding cycle between the spacecraft target and reference source is conditioned by the propagation within 


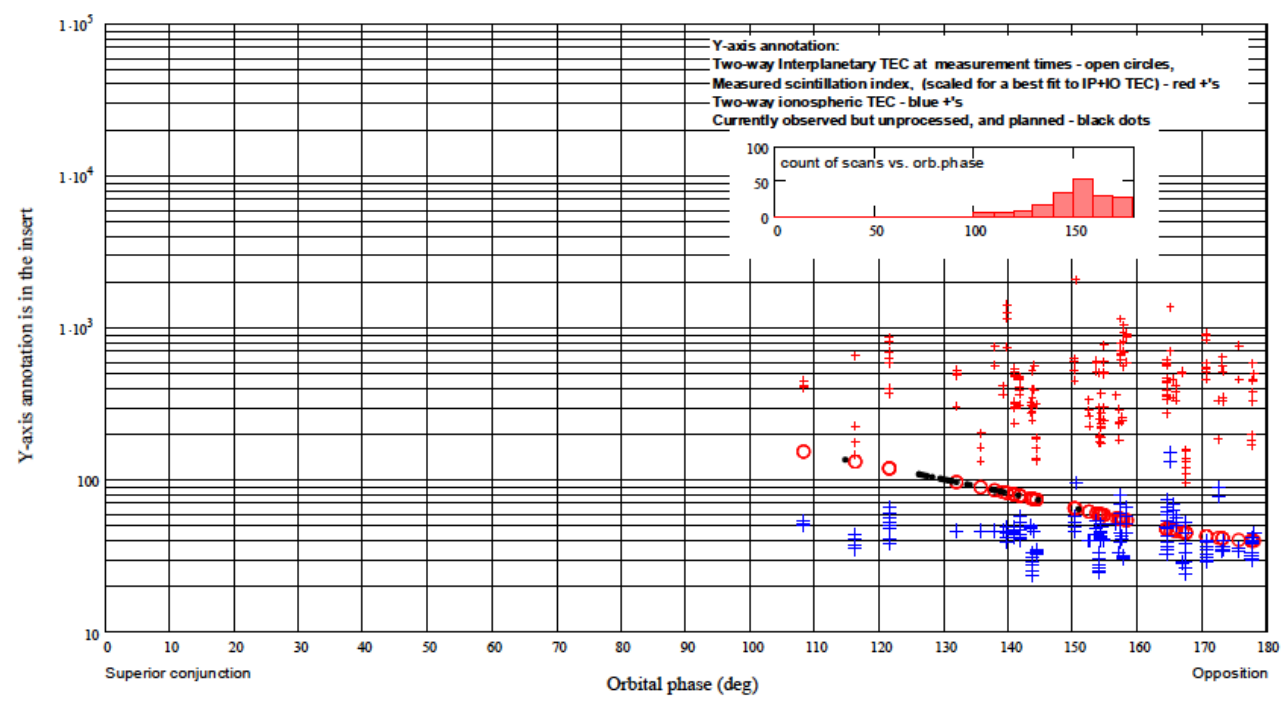

Figure 4: Interplanetary TEC (red circles), ionospheric TEC (brown crosses), Allan variance of the H-maser clocks (black dots), tropospheric contribution (cyan diamonds) and instrumentation error (red line).

the IP. This study allows us to obtain accurate results on scintillation level at any distance and epoch.

This study also allowed us to disentangle and parametrise the contributions of both the interplanetary and Earth's ionospheric TEC's on the spacecraft Doppler detection accuracy with EVN radio telescopes.

The research aims will continue towards characterisation of atmospheric and ionospheric structure of planets and media, small bodies fly-byes, atmospheric drag campaigns, radio occultations or precise orbit determination of satellites.

Tracking observations of MEX spacecraft will continue in order to improve our scintillation measurements. Future space mission, like ESA's Jupiter Icy Moon Explorer (JUICE), will benefit of such precise knowledge.

\section{References}

[Axford, 1968] Axford, W.I. 1968, Reidel, Space Sci. Reviews 8, 331-365

[Duev et al., 2012] Duev, D.A., Molera Calvés, G., Pogrebenko, S.V. et al. 2012, A\&A

[Duev et al., 2015] Duev, D.A., Molera Calvés, G., Pogrebenko, S.V. et al. 2015, A\&A

[Kolmogorov, 1991] Kolmogorov, A.N. 1991, Proc. R. Soc. London, Ser. A, 434, 9

[Molera Calvés, 2012] Molera Calvés G. 2012, PhD dissertation, Aalto University, Pub. No. 42/2012

[Molera Calvés et al., 2014] Molera Calvés G., Cimò, G., Pogrebenko, S.V. \& et al., 2014, A\&A

[Rosenblatt et al., 2012] Rosenblatt, P., Bruinsma, S. L., Müller-Wodarg et al., Icarus, 2012, 217, 831-838

[You et al., 2012] You, X.P., Coles, W.A., Hobbs, G.B., et al. 2012, MNRAS, Vol. 422, 1160-1165 